
\title{
R Reserach S Suare \\ The Study of Boron-Nitride Nanotube Behavior as an Atomic Nano-Pump for Biomedicine Applications
}

\section{Roozbeh Sabetvand}

Amirkabir University of Technology

Hesamodin Jami ( $\nabla$ Hessam.jami@sydney.edu.au )

The University of Sydney

\section{Research Article}

Keywords: Nanopumping, Boron-Nitride Nanotube, Fullerene, Drug Delivery, Molecular Dynamics

Posted Date: June 10th, 2021

DOI: https://doi.org/10.21203/rs.3.rs-163210/v1

License: (c) (i) This work is licensed under a Creative Commons Attribution 4.0 International License. Read Full License

Version of Record: A version of this preprint was published at Journal of Molecular Modeling on December 28th, 2021. See the published version at https://doi.org/10.1007/s00894-021-04990-z. 


\title{
The Study of Boron-Nitride Nanotube Behavior as an Atomic Nano-Pump for Biomedicine Applications
}

\author{
Roozbeh Sabetvand ${ }^{1}$, Hesamodin $\mathrm{Jami}^{2, *}$ \\ ${ }^{1}$ Department of Energy Engineering and Physics, Faculty of Condensed Matter Physics, Amirkabir \\ University of Technology, Tehran, Iran \\ ${ }^{2}$ School of Aerospace, Mechanical and Mechatronic Engineering, The University of Sydney, Sydney, \\ Australia \\ *Corresponding author: Hesamodin Jami, School of Aerospace, Mechanical and Mechatronic \\ Engineering, The University of Sydney, NSW, 2006, Australia \\ E-mail: hessam.jami@sydney.edu.au
}

\begin{abstract}
In this study we use molecular dynamics (MD) simulations to describe the nanopumping process of Boron Nitride Nanotube (BNNT) with fullerene molecule displacement for the first time. Technically, for the simulation of BNNT and fullerene structures, we used Tersoff force-field. The result of the equilibrium process of these structures shows the excellent stability of them which this atomic behavior arises from the appropriate settings in our MD simulations. Further, to describe the BNNT nanopumping process, we calculate the velocity and translational/rotational kinetic energy of fullerene molecule. Numerically, by increasing of simulated structures temperature from $275 \mathrm{~K}$ to $350 \mathrm{~K}$, the nanopumping time varies from 9.31 ps to $8.55 \mathrm{ps}$, respectively. Further, the atomic wave producing in BNNT is an important parameter for nanopumping process and we decrease the nanopumpint time to 7.79 ps by this atomic parameter optimization.
\end{abstract}

Keywords: Nanopumping, Boron-Nitride Nanotube, Fullerene, Drug Delivery, Molecular Dynamics.

\footnotetext{
Nomenclature

$\mathrm{F}_{\mathrm{ij}}, \quad$ interatomic force between atoms i and $\mathrm{j}(\mathrm{eV} / \AA)$;

$\mathrm{V}_{\mathrm{ij}}, \quad$ interatomic potential $(\mathrm{eV})$;

$\mathrm{m}$, atomic mass $(\mathrm{u})$;

$\mathrm{r}_{\mathrm{ij}}, \quad$ atomic distance between atoms $\mathrm{i}$ and $\mathrm{j}(\AA)$;
} 
t, $\quad$ simulation time (ps);

v atomic velocity $(\AA / p s)$;

$\mathrm{f}, \quad$ frequency of atomic oscillation $(1 / \mathrm{ps})$;

$\mathrm{A}_{0}, \quad$ amplitude of atomic oscillation $(\AA)$;

$\mathrm{R}$, distance parameter in Tersoff potential $(\AA)$;

D, distance parameter in Tersoff potential $(\AA)$;

A, energy parameter in Tersoff potential $(\mathrm{eV})$;

B, energy parameter in Tersoff potential $(\mathrm{eV})$;

\section{Greek symbols}

$\lambda_{1}, \quad$ distance parameter in Tersoff potential (1/Å);

$\lambda_{2}$, distance parameter in Tersoff potential (1/Å);

$\Delta \mathrm{t}, \quad$ molecular dynamics time step;

\section{Introduction}

Nanotechnology is the manipulation of structures on a nanometric scale. The first widespread definition of this technology referred to the particular practical goal of optimizing atoms for production of macro-scale applications [1-2]. Boron-Nitride Nanotube (BNNT) is one of the promising materials for various nanotechnology aims [34]. These nanostructures are a polymorph of boron nitride mixture. Historically, BNNTs were introduced in 1994 and produced in 1995 for the first time [5]. Structurally, these nanostructures are similar to common carbon nanotubes (CNTs), which are atomic cylinders with nanometric size, except that $\mathrm{C}$ atoms are substituted by $\mathrm{N}$ and $\mathrm{B}$ atoms [68]. However, the physical behavior of BN nanotubes are different. BNNTs have attractive properties, when compared to CNTs, providing an extensive range of scientific and industrial applications [9]. Mechanically, BNNTs and CNTs have similar strength properties of circa 100-times stronger than steel [10]. So this material can be used to transfer of nanostructures. Further, toxicological researches on BNNTs conducted in the recent years seem to show that the enhanced chemical inertia of BNNTs favors biocompatibility. As a result, their use in the medical aims [11-14]. For example, these nanostructures have been used as a nanometric apparatus for drug delivery process and formed the basis of the new branch in situ human organ pressure detection [15-16]. In previous researches, $\mathrm{Xu}$ et al. [17] reported that drug structures could bind to the BNNTs surface stably with atomic interactions. The drugs can then be delivered directly into the 
cells for cancer treatment. Shayan et al. [18] described that the physical properties of the armchair BNNTs interacted with the 5-FU drug, as an anticancer drug, at the B3LYP/6$31 \mathrm{G}(\mathrm{d}, \mathrm{p})$ level of theory. The results of this study show that the encapsulation and adsorption of 5-FU molecule on the BNNTs surface are favorable processes. Khatti et al. [19] investigated a drug delivery system based on BNNTs, particularly the delivery of platinum-based anticancer drugs. For this propose, the behavior of carboplatin drugs inserted in BNNT as a carrier was studied. The results of this report show promising behavior of BNNT structure for delivery of platinum-based drugs. Technically, nanopumping process is one of the common ways to drug delivery procedure in various nanotubes. Atomic pumping process can be done effectively by BNNT structure because of the van der Waals interaction between external atoms and this structure. In this nanoscale mechanism, the inserted atoms to BNNT would prefer to stay inside this nanotube [20-23]. Physically, there is an energy barrier preventing an encapsulated inserted atoms inside an atomic structure from coming out of the nanotube [24]. Today, researchers need to introduce an effective actuation mechanism to eject the atoms out of the BNNT for various aims such as biomedicine applications. Molecular Dynamics (MD) method is one of the important methods in the study of various nanostructures behavior [25-28]. Theoretically, nanostructures consist of a vast number of atoms, and it is impossible to estimate the properties of such computationally large structures, analytically. Computational methods such as MD simulations used for this purpose. In other researches, this computational method used for study of CNTs nanopumping process [29-30]. However, BNNTs nanopumping behavior don't reported in previous MD reports. In this work, we used this computational approach to study of nanopumping process of BNNT structure. For this, we propose to actuate wave propagation in a BNNT by $\mathrm{Cu}$ (Copper) oscillating tips and demonstrate the nanopumping process of a $\mathrm{C}_{20}$ molecule (fullerene) via an ideal BNNT.

\section{Computational Method}

In this computational work, we used Large Scale Atomic/Molecular Passively Simulator (LAMMPS) to demonstrate the BNNT performance as nanopumping structure [31-32]. In 
other words, by using this MD simulation package, BNNT and $\mathrm{C}_{20}$ atomic structures simulated as figure 1. Computationally, in depicted structure, periodic boundary conditions implemented in $\mathrm{x}$-direction and fix one used for $\mathrm{y}$ and $\mathrm{z}$ directions [33]. Next, Nose-Hoover thermostat used to temperature equilibrates of the simulated system [34-35]. This computational thermostat equilibrates the BNNT and $\mathrm{C}_{20}$ temperature at $300 \mathrm{~K}$ with 0.1 damping rate.

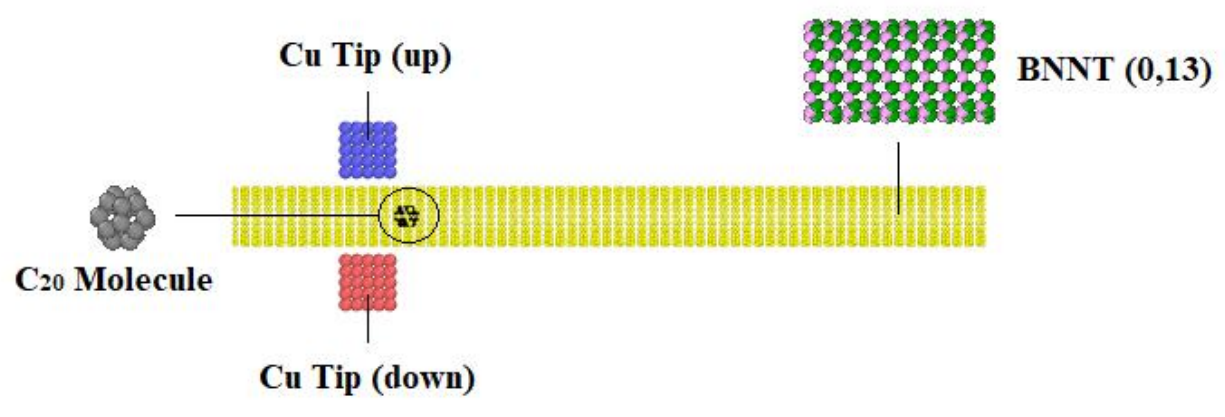

Figure. 1. Schematic of BNNT, $\mathrm{C}_{20}$ and $\mathrm{Cu}$ tips arrangement in MD simulation box.

Interatomic force-field is a significant parameter in MD simulations. To simulate the atomic structures in our computational study, we use Tersoff force-field [36-37]. Theoretically, this computational function defined as below [37]:

$E=\frac{1}{2} \sum_{i} \sum_{j \neq i} V_{i j}$

$V_{i j}=f_{C}\left(r_{i j}\right)\left[f_{R}\left(r_{i j}\right)+b_{i j} f_{A}\left(r_{i j}\right)\right]$

Where, $\mathrm{f}_{\mathrm{R}}$ is a two-body term and $\mathrm{f}_{\mathrm{A}}$ includes three-body interactions. The summations in the equation (2) are over all neighbors $\mathrm{j}$ and $\mathrm{k}$ of an atom $\mathrm{i}$ within a cutoff distance. Computationally, $\mathrm{f}_{\mathrm{C}}, \mathrm{f}_{\mathrm{R}}$, and $\mathrm{f}_{\mathrm{A}}$ constants can be expressed as below equations:

$f_{C}(r)=\frac{1}{2}-\frac{1}{2} \sin \left(\frac{\pi}{2} \frac{r-R}{D}\right)$ 
$f_{R}(r)=A \exp \left(-\lambda_{1} r\right)$

$f_{A}(r)=-B \exp \left(-\lambda_{2} r\right)$

in these equations, $\mathrm{R}$ and $\mathrm{D}$ have the distance dimension, $\mathrm{A}$ and $\mathrm{B}$ have the energy dimension, and $\lambda_{1}$ and $\lambda_{2}$ have the $\frac{1}{\text { distance }}$ dimension. These constants value in our MD simulations reported in Table 1.

Table. 1. The Tersoff potential parameters in our MD simulations [37].

\begin{tabular}{|c|c|c|c|c|c|c|}
\hline Interaction Type & $\mathbf{R}(\AA)$ & $\mathbf{D}(\AA)$ & $\mathbf{A}(\mathbf{e V})$ & $\mathbf{B}(\mathbf{e V})$ & $\boldsymbol{\lambda}_{\mathbf{1}}(\mathbf{1} / \AA)$ & $\boldsymbol{\lambda}_{\mathbf{2}}(\mathbf{1} / \AA)$ \\
\hline B-N-C & 1.95 & 0.05 & 1380.0 & 340.00 & 3.568 & 2.199 \\
\hline
\end{tabular}

Further, $\mathrm{Cu}$ atoms interaction described by Embedded Atom Model (EAM) which defined as below [38-39]:

$$
E_{i}=F_{\alpha}\left(\sum_{i \neq j} \rho_{\beta}\left(r_{i j}\right)\right)+\frac{1}{2} \sum_{j \neq i} \varphi_{\alpha \beta}\left(r_{i j}\right)
$$

Where, $F$ is the embedding energy which is a function of the atomic electron density $\rho, \varphi$ is a pair potential interaction, and $\alpha$ and $\beta$ are the element types of atoms $i$ and $j$. Finally, the atomic interaction between defined structures described with Lennard Jones function by using DREIDING information for various elements [40]. After determining interatomic force-fields to various structures, MD simulation process was fulfilled. Theoretically, to describe the atoms time evolution in the simulation box, Newton's law is used as the gradient of force-field function [41]:

$$
\begin{aligned}
F_{i} & =\sum_{i \neq j} F_{i j}=m_{i} \frac{d^{2} r_{i}}{d t^{2}}=m_{i} \frac{d v_{i}}{d t} \\
F_{i j} & =-\operatorname{grad} V_{i j}
\end{aligned}
$$

In equations (7) and (8), integrate of the Newton law is done by the Velocity-Verlet algorithm as below [42-43]:

$$
r(t+\Delta t)=r(t)+v(t) \Delta t+\frac{1}{2} a(t) \Delta t^{2}+O\left(\Delta t^{4}\right)
$$




$$
v(t+\Delta t)=v(t)+\frac{a(t)+a(t+\Delta t)}{2} \Delta t+O\left(\Delta t^{2}\right)
$$

Where $r(t+\Delta t)$ and $v(t+\Delta t)$ is the atomic position and velocity in $t+\Delta t$, respectively. Finally, according to the reported descriptions, MD simulations in this computational study carried out in below main steps:

Step A: Initial BNNT/C 20 and $\mathrm{Cu}$ tips simulated with Tersoff and EAM interatomic forcefields, respectively. After this simulation process, atomic structures equilibrated by NoseHoover thermostat for $10 \mathrm{~ns}$. For this purpose, MD simulation temperature set at $300 \mathrm{~K}$ and the atomic behavior of atomic mixtures reported by the temperature and potential energy of them.

Step B: Next, BNNT nanopumping process simulated by $\mathrm{Cu}$ tips oscillation. For nanopumping process study, physical parameters such as velocity, transitional/rotational component of kinetic energy for $\mathrm{C}_{20}$ molecule reported. In this step of our computational study, the effect of: a) MD simulation temperature and b) amplitude/frequency variation of $\mathrm{Cu}$ tips oscillations on BNNT nanopumping process reported.

\section{Results and Discussion}

\subsection{Equilibration Process of Atomic Structures}

In the first step, the equilibrium process of simulated structures including BNNT, $\mathrm{C}_{20}$ and Cu tips studied at initial temperatures ( $\mathrm{T}=275 \mathrm{~K}, 300 \mathrm{~K}, 325 \mathrm{~K}$, and $350 \mathrm{~K})$. Our MD results show that, the atoms arrangement in the simulated system adopted with defined forcefields. This physical phase of structures reported by the temperature and potential energy calculation. From figure 2, we can say the simulated structures temperature changes as a function of simulation time and converged to initial temperature after $1 \mathrm{~ns}$. Physically, this physical equilibrium arises from atomic oscillation reducing over MD simulation time. Furthermore, figure 3 shows the potential energy of atomic structures as a function of MD simulation time. From this figure, the potential energy converged to $-9224 \mathrm{eV}$ after 10000000 -time steps at $\mathrm{T}=275 \mathrm{~K}$. By temperature increasing, the atomic structures don't 
disrupt and equilibration phase can be detected after $10 \mathrm{~ns}$. By further analyzing, we conclude the atomic stability of simulated structures decreases by temperature changes from $\mathrm{T}=300 \mathrm{~K}$ to $\mathrm{T}=350 \mathrm{~K}$. Numerically, by temperature increasing, the potential energy decreases to $-9218 \mathrm{eV},-9213 \mathrm{eV}$ and $-9208 \mathrm{eV}$ which this atomic behavior arises from atomic fluctuations in MD simulation box. Physically, by atomic fluctuation increasing, the mean distance between various atoms increases and by this phenomenon occur, potential energy/atomic stability decreases.

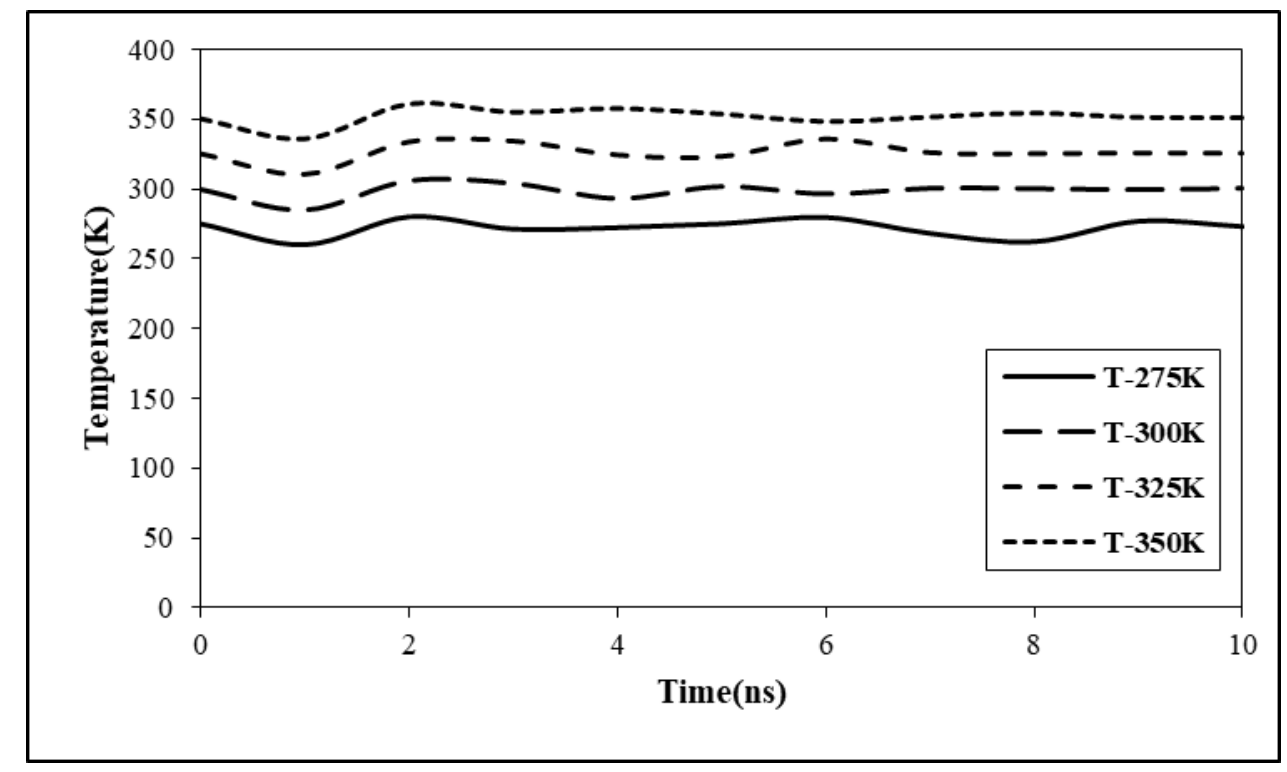

Figure 2. Temperature variation of $\mathrm{BNNT}, \mathrm{C}_{20}$ and $\mathrm{Cu}$ tips as a function of $\mathrm{MD}$ simulation time. 


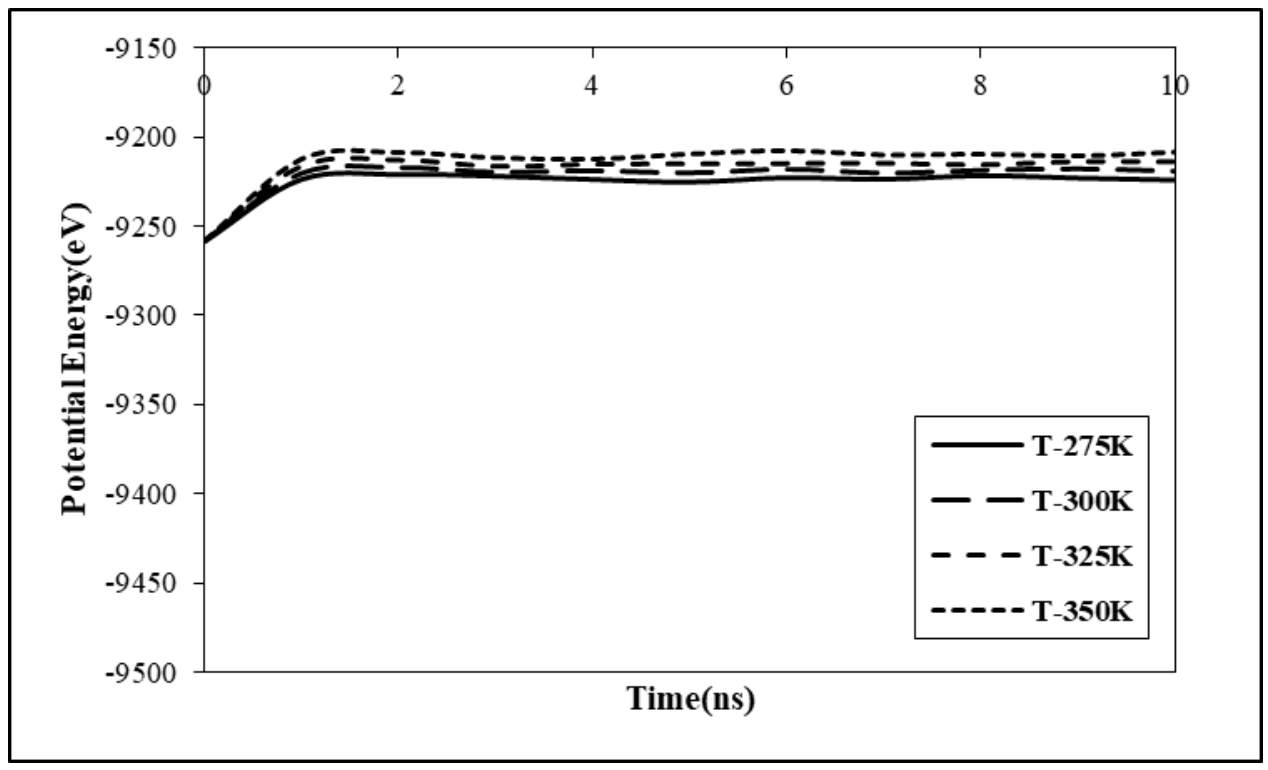

Figure 3. Potential energy variation of $\mathrm{BNNT}, \mathrm{C}_{20}$ and $\mathrm{Cu}$ tips as a function of MD simulation time.

\subsection{Nanopumping Process in BNNT Structure}

In this step, pristine atomic arrangement improved by two $\mathrm{Cu}$ tips (up and down) oscillation for nanopumping process simulation. Computationally, for BNNT nanopumping process describing, velocity and kinetic energy of $\mathrm{C}_{20}$ molecule reported. Figure 4 shows the time evolution of simulated structure after $10 \mathrm{ps}$. This atomic behavior arises from $\mathrm{Cu}$ tips oscillation with $1.75 \AA$ A magnitude and $0.50 \mathrm{THz}$ frequency in the below equations:

$\mathrm{Y}_{\text {top }}=\mathrm{A}_{0} \sin (2 \pi \mathrm{ft})$

$\mathrm{Y}_{\text {down }}=-\mathrm{A}_{0} \sin (2 \pi \mathrm{ft})$

As shown in figure 4, the fullerene molecule is inserted inside next to the atomic tips at the end of the nanotube. As the $\mathrm{Cu}$ tips oscillate, these structures actuated wave push the fullerene molecule to move in z-direction due to the repulsive interaction between the $\mathrm{C}_{20}$ molecule and the BNNT atoms. Numerically, the position of $\mathrm{C}_{20}$ molecule varies from $0 \AA$ to $100 \AA$ in z-direction after 8.99 ps. This atomic behavior comparable with previous theoretical works and show the correctness of our MD simulation method [44-45]. 


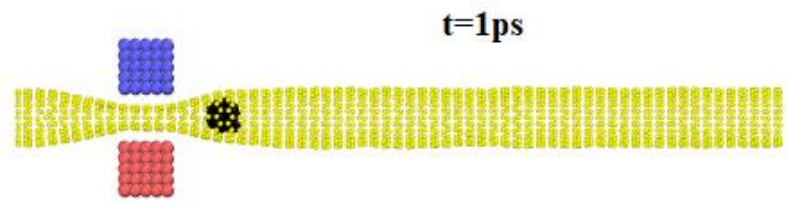

a

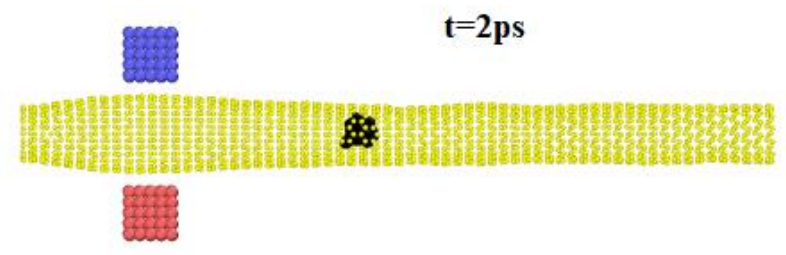

b

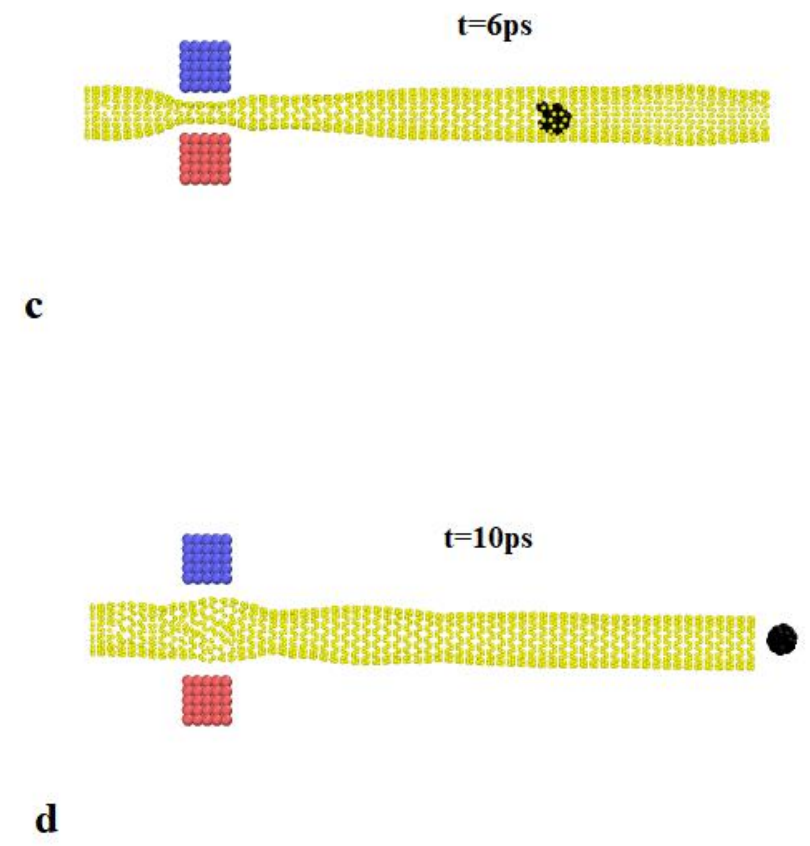


Figure 4. Time evolution of nanopumping process in BNNT at a) $t=1 p s, b) t=4 p s, c) t=6 p s$, and d) $t=10$ ps.

Theoretically, from the time evolution of $\mathrm{C}_{20}$ molecule inside BNNT, we conclude the MD simulation time is long enough to the nanopumping process occur. After this atomic procedure, the velocity and kinetic energy of fullerene reported in figures 5 and 7 , respectively. The atomic velocity of fullerene shows that, the atomic waves are increased by simulation time passing, and push fullerene in the direction of the wave propagation. Numerically, the $\mathrm{C}_{20}$ molecules reach to the right end of BNNT after 8.99 ps. Finally, the carbon structure ejected out of the BNNT and nanopumping process completed after 0.32 ps later (9.31 ps). Physically, nanopumping process consists of 2 main steps to push the fullerene inside the BNNT. These steps can be described by calculating the $\mathrm{C}_{20}$ molecule velocity as a function of MD simulation time and temperature, as depicted in figure 5. From our calculations at $\mathrm{T}=275 \mathrm{~K}$, we conclude the maximum value of $\mathrm{C}_{20}$ acceleration occurs in the 0 to 734 -time steps, and this molecule velocity reaches to $1211 \mathrm{~m} / \mathrm{s}$. After this atomic phase, the $\mathrm{C}_{20}$ molecule continues to accelerate at a lower value for the 4200-time steps and velocity value of fullerene reach to $1682 \mathrm{~m} / \mathrm{s}$. In the next phase, fullerene velocity gradually reduced to $1375 \mathrm{~m} / \mathrm{s}$ upon to right end of the BNNT before being ejected out of the nanotube. So in nanopumping process, the fullerene velocity first increases and then decreases which this atomic behavior arises from interatomic bonds between $\mathrm{C}_{20}$ molecule and BNNT. Despite the $\mathrm{C}_{20}$ velocity decreasing, this structure has sufficient value of kinetic energy to overcome the energy barrier of the atomic nanotube end. Numerically, the fullerene molecule speed after the ejection process is $448 \mathrm{~m} / \mathrm{s}$ which this atomic value can be important for biomedical applications such as drug delivery process. 


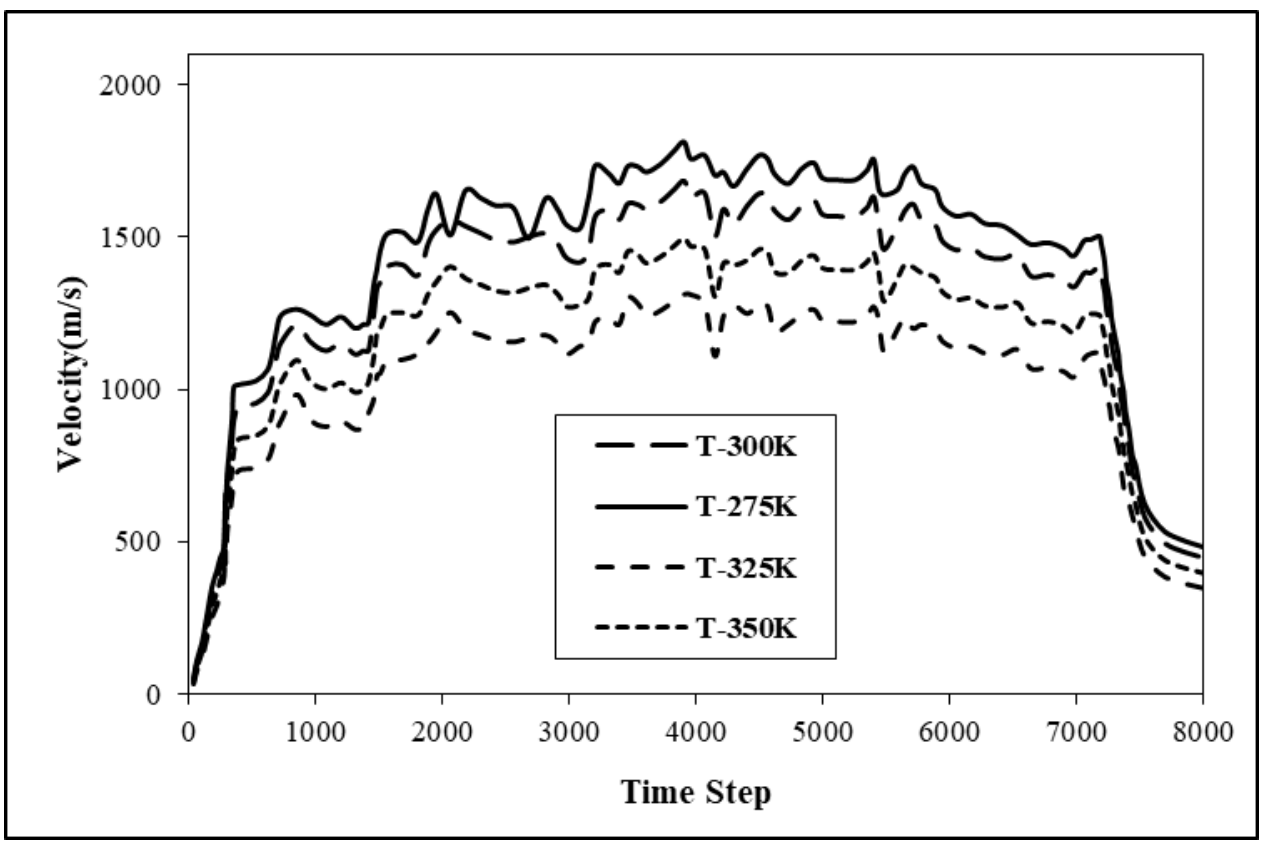

Figure 5. Velocity of $\mathrm{C}_{20}$ molecule during the nanopumping process as a function of simulation time steps in various initial temperature.

Further, our MD simulation results showed the $\mathrm{C}_{20}$ molecules has a rotational motion in nanopumping process, and this atomic structure simultaneously rotates as it translates in the z-direction. To observe the rotational motion of fullerene inside the BNNT structure, images of $\mathrm{C}_{20}$ displacement depicted in figure 6 . So, through the interaction between carbon atoms and BNNT, the nanotube wave transfers the energy into the fullerene in translational/rotational components as depicted in figure $7(\mathrm{~T}=275 \mathrm{~K})$. We conclude, when the fullerene rotates with high velocity, the translational motion along the $\mathrm{z}$-direction disrupted which this atomic behavior arises from translational velocity decreasing. Thus, in biomedicine applications such as drug delivery, it is crucial to minimize the rotational energy (rotational velocity) of target material in order to increase translational energy and to increase the nanopumping efficiency. 


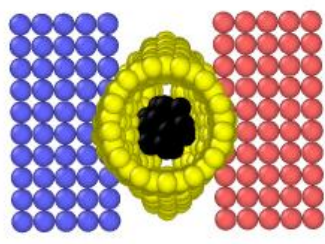

$\mathbf{t}=1 \mathrm{ps}$

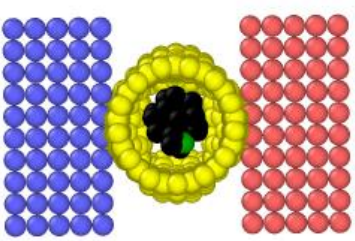

$\mathbf{t}=2 \mathrm{ps}$

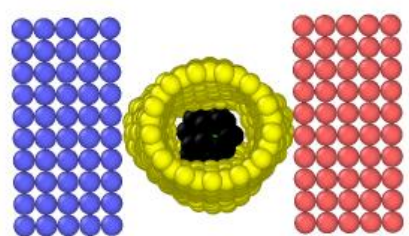

$\mathbf{t}=\mathbf{3}$ ps

Figure 6. Evolution of $\mathrm{C}_{20}$ molecule in BNNT as a function of simulation time in successful nanopumping process (side view).

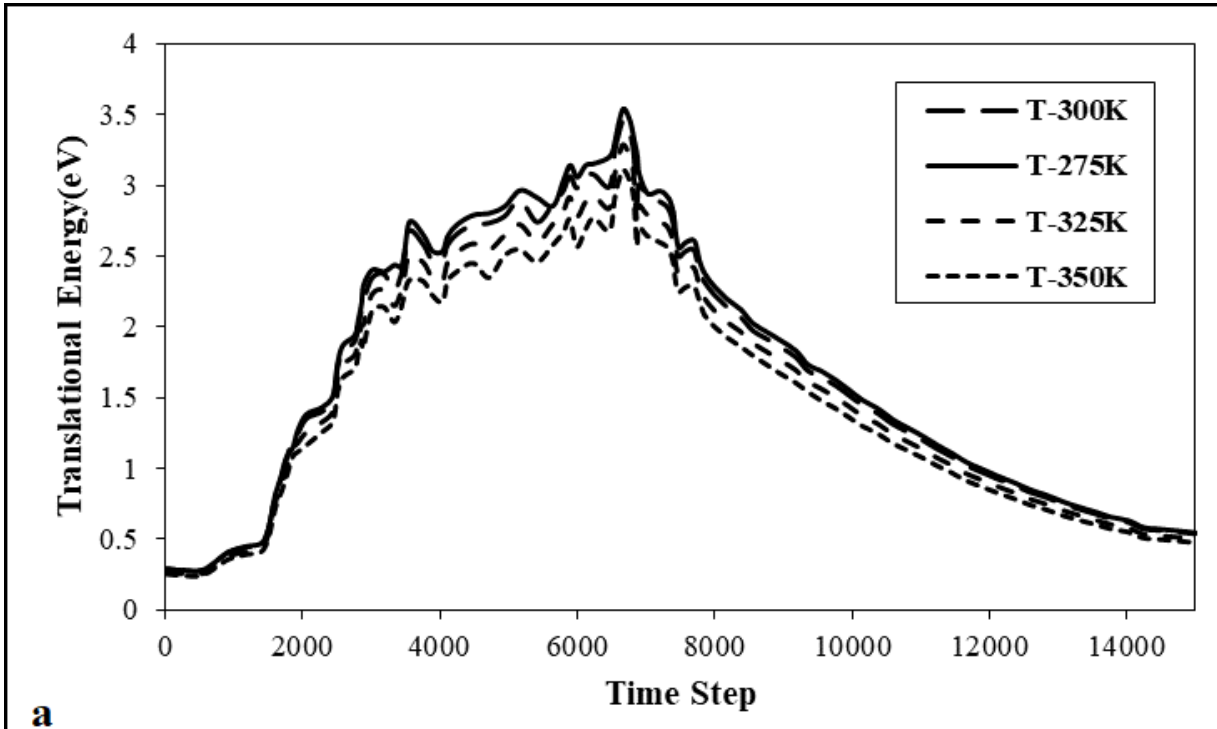




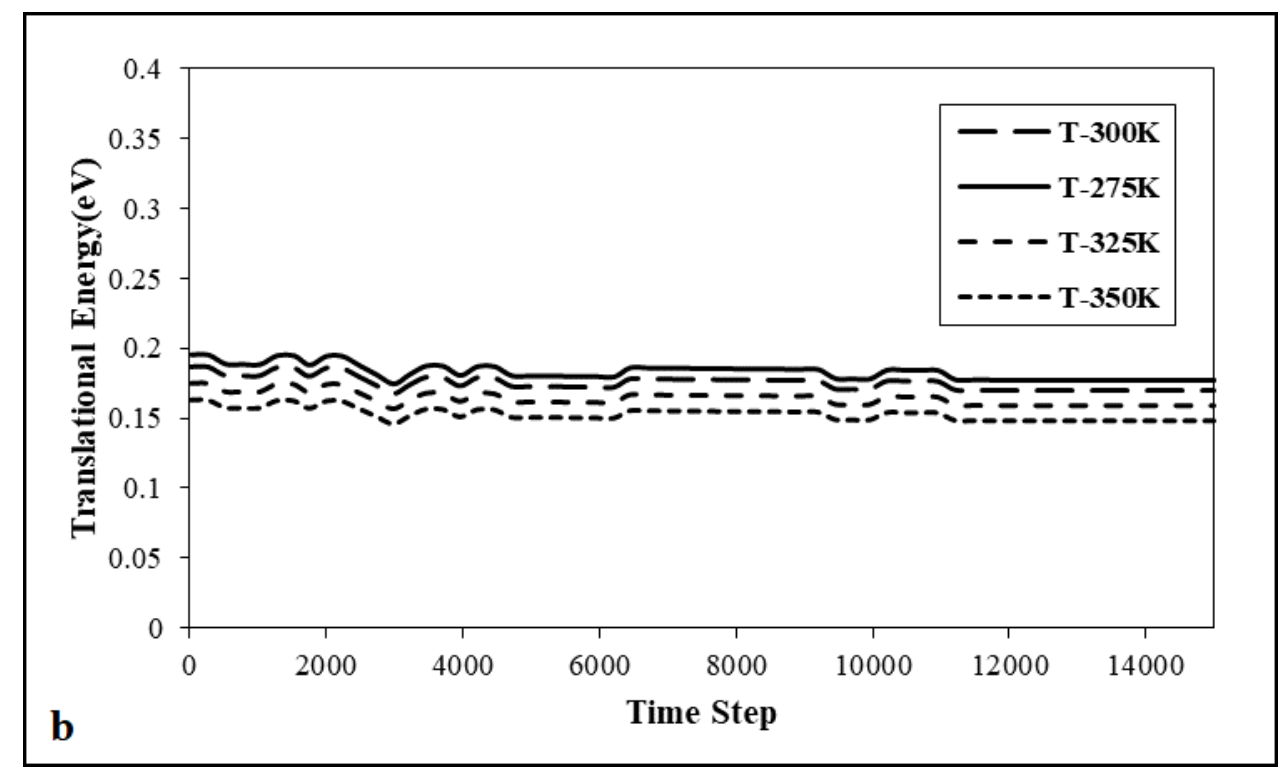

Figure 7. Comparison of a) translational and b) rotational component of $\mathrm{C}_{20}$ kinetic energy in successful pumping process for various initial temperatures.

As shown in figure 8 , the temperature of simulated structures is an important parameter in nanopumping process. For describe of this parameter effects on BNNT nanopumping process, the initial temperature of structures set to $\mathrm{T}=275 \mathrm{~K}, \mathrm{~T}=300 \mathrm{~K}, \mathrm{~T}=325 \mathrm{~K}$, and $\mathrm{T}=350 \mathrm{~K}$. Physically, by temperature increasing the translational velocity and so, nanopumping efficiency decreased. This atomic behavior arises from atomic fluctuations increasing by temperature changes from $275 \mathrm{~K}$ to $350 \mathrm{~K}$. Numerically, the temperature increases to $350 \mathrm{~K}$ the nanopumping process occur after $9.31 \mathrm{ps}$. By more temperature increasing and reach to $400 \mathrm{~K}$, the nanopumping process disrupted and this atomic process get unsuccessful (see figure 8). Thus we conclude, the nanopumping process delayed with temperature increasing. The numerical results of MD simulations in this step of our study reported in Table 2.

Table 2. The maximum value of translational/rotational kinetic energy and translational velocity of fullerene as a function of MD simulation temperature.

\begin{tabular}{|c|c|c|c|}
\hline Temperature(K) & $\begin{array}{c}\text { Transitional Energy } \\
\operatorname{Maximum}(\mathrm{eV})\end{array}$ & $\begin{array}{c}\text { Rotational Energy } \\
\text { Maximum }(\mathrm{eV})\end{array}$ & $\begin{array}{c}\text { Translational Velocity } \\
\text { Maximum }(\mathbf{m} / \mathbf{s})\end{array}$ \\
\hline
\end{tabular}




\begin{tabular}{|c|c|c|c|}
\hline 275 & 3.65 & 0.222 & 1871 \\
\hline 300 & 3.41 & 0.190 & 1708 \\
\hline 325 & 3.02 & 0.185 & 1431 \\
\hline 350 & 3.94 & 0.181 & 1202 \\
\hline
\end{tabular}

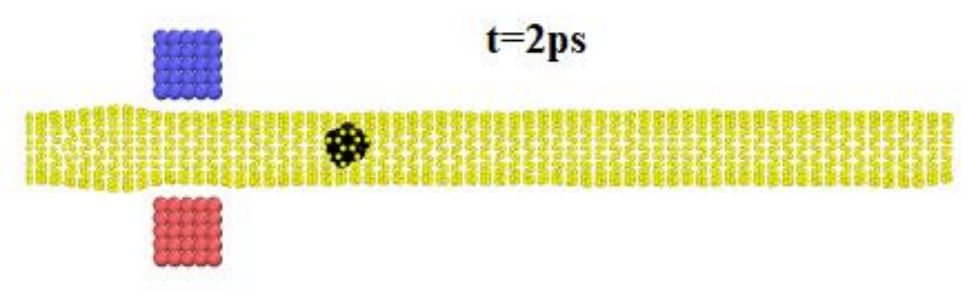

a

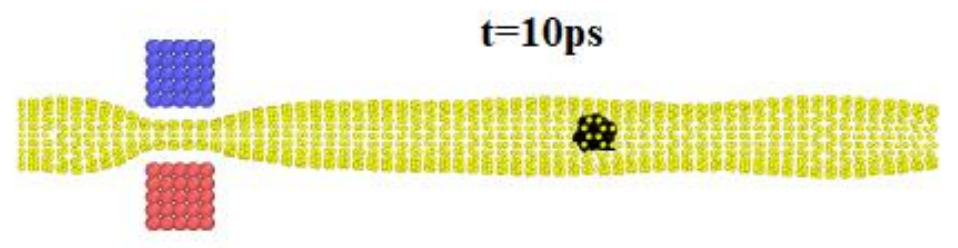

b

Figure 8. BNNT and fullerene atomic positions in a) $t=2$ ps and $b$ ) $t=10$ ps (final step) of unsuccessful nanopumping process at $\mathrm{T}=400 \mathrm{~K}$.

Because the efficiency of nanopumping can be manipulated with $\mathrm{Cu}$ tips oscillation, amplitude and frequency of up and down tips optimized in the final step of this work. We simulate the nanopumping process for various $\mathrm{Cu}$ tips amplitude and frequencies which some of the MD simulation results reported in figure 9. Our MD simulations show the 1.75 
$\AA$ amplitude of oscillation for BNNT is appropriate value in nanopumping process. Physically, if the $\mathrm{Cu}$ atoms oscillation amplitude get too large ( $\mathrm{A}>2.75 \AA$ ), it causes the upper and lower regions of the BNNT to form bond and the $\mathrm{Cu}$ tips oscillation leading to breakdown of actuation. Further, the optimized value for $\mathrm{Cu}$ tips oscillation frequency is $0.75 \mathrm{THz}$. Our results show that, if the $\mathrm{Cu}$ tips oscillation frequency get lower than 0.50 $\mathrm{THz}, \mathrm{C}_{20}$ molecule exhibits large rotational energy and nanopumping process get unsuccessful. If the frequency get higher than $2.25 \mathrm{THz}$, no effective atomic wave can be detected in the BNNT, and it takes a long time to complete the nanopumping process. Finally, our MD results show the $\mathrm{Cu}$ tips oscillation with $\mathrm{A}=1.75 \AA$ amplitude and $\mathrm{f}=0.75$ $\mathrm{THz}$ frequency is optimized values for nonpumping process in BNNT as depicted in figure 10 .

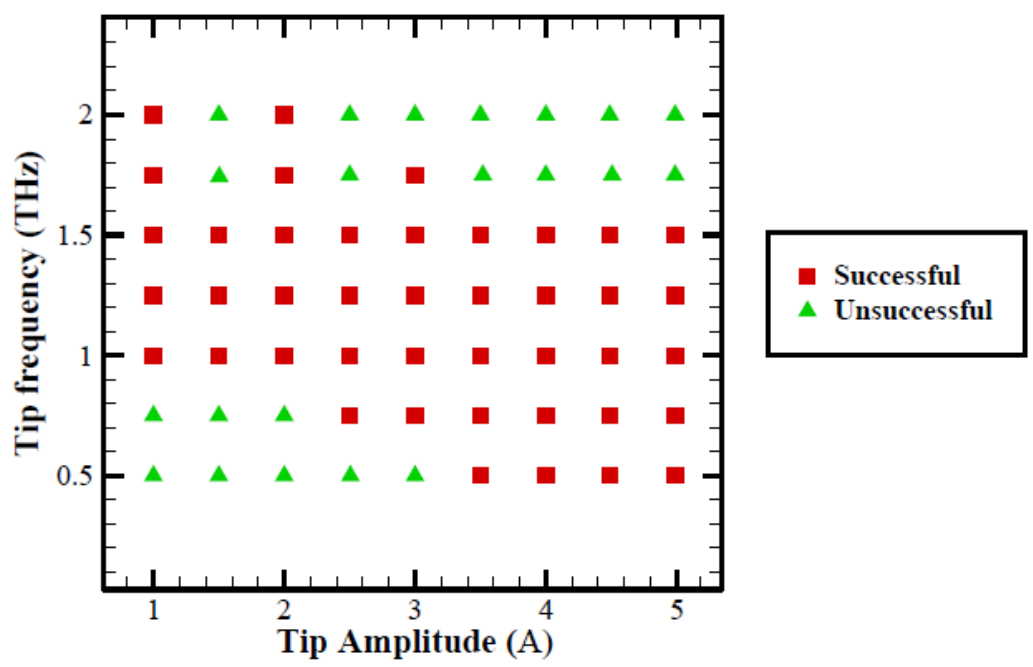

Figure 9. Successful and unsuccessful nanopumping process for the various value of atomic tips amplitude and frequency. 

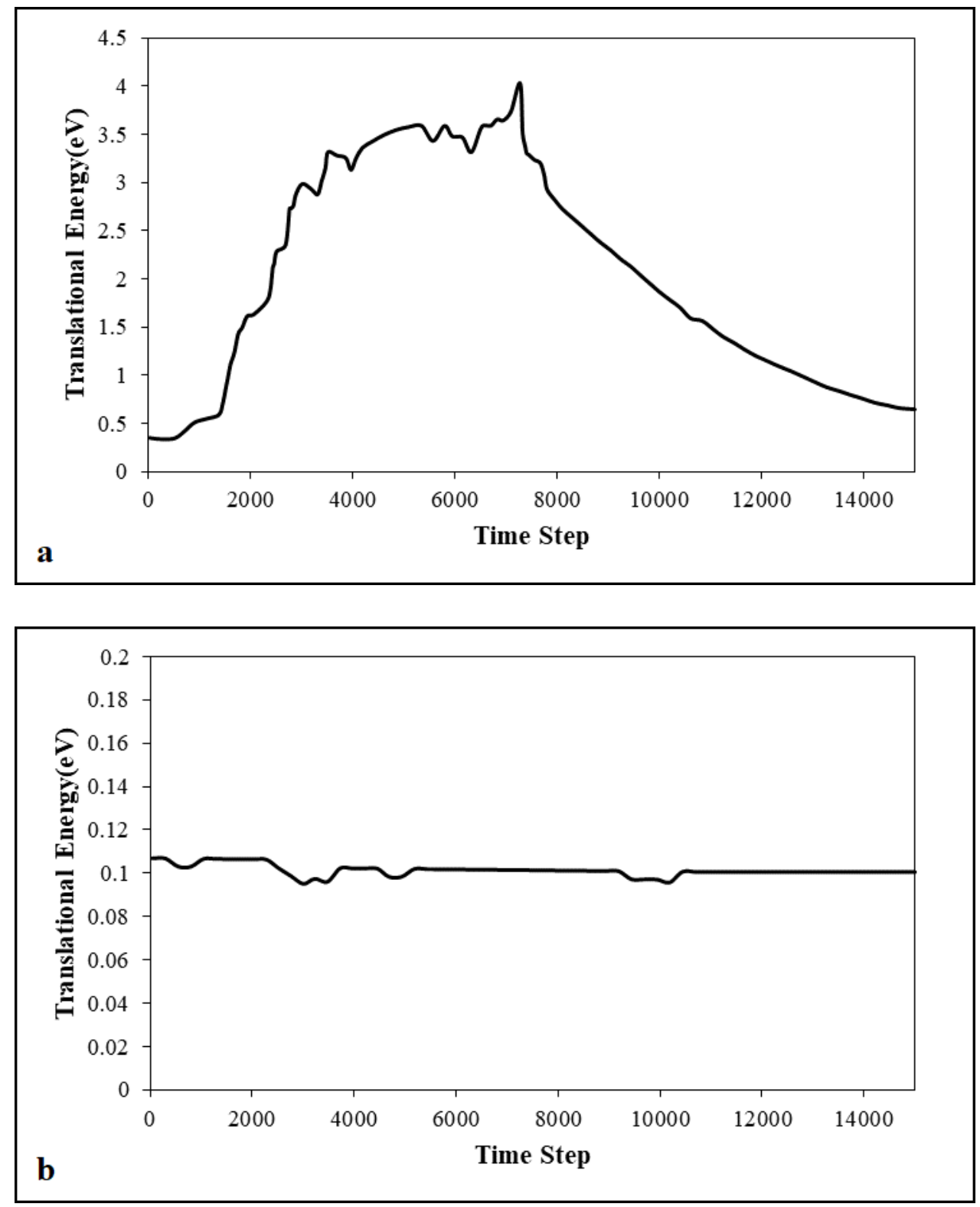

Figure 10. Comparison of the a) translational and b) rotational components of $C_{20}$ molecule kinetic energy in optimum condition for BNNT nanopumping process.

\section{Conclusion}

In this study, we use molecular dynamics (MD) method to describe the nanopumping process in Boron-Nitride nanotube (BNNT) structure. Technically, this atomic process detected by $\mathrm{C}_{20}$ molecule time evolution in various temperature. Our computational results from MD simulations are as following: 
A. Embedded Atom Model (EAM) and Tersoff interatomic force-fields are the appropriate functions to MD simulation of BNNT nanopumping process.

B. By increasing the temperature of simulated structures from $275 \mathrm{~K}$ to $350 \mathrm{~K}$, the nanopumping process occurs in larger simulation time.

C. Temperature variation is an important parameter in nanopumping process and this atomic procedure can be disrupted by a large increase in initial temperature.

D. Numerically, the CNT nanopumping process optimized by using $1.75 \AA$ and 0.75 $\mathrm{THz}$ values for amplitude and frequency of atomic tips oscillation.

E. Atomic tips frequency changes can be disrupted of nanopumping process. Numerically, this atomic process failed for $\mathrm{f}>2.25 \mathrm{THz}$.

F. Atomic tips amplitude changes can be disrupted of nanopumping process. Numerically, this atomic process failed for A>2.75 A.

These MD simulation results were shown the physical parameters such as: atomic temperature and amplitude/frequency of $\mathrm{Cu}$ tips oscillation are essential in BNNT nanopumping process and by these parameters optimization, nanopumping process of BNNT can be enhanced considerably.

\section{Declarations}

Funding The authors received support from High Performance Computing Research Center of Amirkabir University of Technology (Tehran Polytechnic).

Conflicts of interest/Competing interests The authors declare that they have no conflicts of interest.

Ethics approval N/A

Consent to participate N/A

Consent for publication N/A

Availability of data and material Data available on request from the authors.

Code availability LAMMPS main input (MD simulation input) available on request from the authors.

Authors' contributions Roozbeh Sabetvand: Designed the analysis, Performed the analysis; 
Wrote the paper. Hesamodin Jami: Designed the analysis, Proof reading.

\section{References}

[1] Golberg, Dmitri; Costa, Pedro M. F. J.; Mitome, Masanori; Bando, Yoshio (2009). "Properties and engineering of individual inorganic nanotubes in a transmission electron microscope". Journal of Materials Chemistry. 19 (7): 909. doi:10.1039/B814607A.

[2] Rubio, A.; et al. (1994). "Theory of Graphitic Boron Nitride Nanotubes". Physical Review B. 49 (7): 5081-5084. Bibcode:1994PhRvB..49.5081R. doi:10.1103/PhysRevB.49.5081. PMID 10011453.

[3] Chopra, N. G.; et al. (1995). "Boron Nitride Nanotubes". Science. 269 (5226): 966-7. Bibcode:1995Sci...269..966C. doi:10.1126/science.269.5226.966. PMID 17807732.

[4] Blase, X.; et al. (1994). "Stability and Band Gap Constancy of Boron Nitride Nanotubes". Europhysics Letters (EPL). 28 (5): 335. Bibcode:1994EL....28..335B. doi:10.1209/02955075/28/5/007. S2CID 120010610.

[5] Wei-Qiang Han; et al. (2002). "Transformation of BxCyNz Nanotubes to Pure BN Nanotubes" (PDF). Applied Physics Letters. 81 (6): 1110. Bibcode:2002ApPhL..81.1110H. doi:10.1063/1.1498494.

[6] Golberg, D.; Bando, Y.; Tang, C.C. \& Zhi, C.Y. (2007). "Boron Nitride Nanotubes". Advanced Materials. 19 (18): 2413. doi:10.1002/adma.200700179.

[7] Ciofani, Gianni; Danti, Serena; Genchi, Giada Graziana; Mazzolai, Barbara; Mattoli, Virgilio (2013-05-27). "Boron Nitride Nanotubes: Biocompatibility and Potential Spill-Over in Nanomedicine". Small. 9 (9-10): 1672-85. doi:10.1002/smll.201201315. PMID 23423826.

[8] Şen, Özlem; Emanet, Melis; Çulha, Mustafa (2016-01-01), Ciofani, Gianni; Mattoli, Virgilio (eds.), "Chapter 3 - Biocompatibility evaluation of boron nitride nanotubes", Boron Nitride Nanotubes in Nanomedicine, Micro and Nano Technologies, William Andrew Publishing, pp. 4158, ISBN 978-0-323-38945-7, retrieved 2020-03-20.

[9] Kim, Jun Hee; Pham, Thang Viet; Hwang, Jae Hun; Kim, Cheol Sang; Kim, Myung Jong (2018-06-28). "Boron nitride nanotubes: synthesis and applications". Nano Convergence. 5 (1): 
17. Bibcode:2018NanoC...5...17K. doi:10.1186/s40580-018-0149-y. ISSN 2196-5404. PMC 6021457. PMID 30046512.

[10] Ju, S.-P., Wang, Y.-C., \& Lien, T.-W. (2011). Tuning the electronic properties of boron nitride nanotube by mechanical uni-axial deformation: a DFT study. Nanoscale Research Letters, 6(1), 160. doi:10.1186/1556-276x-6-160.

[11] Lee, C. H.; Qin, S.; Savaikar, M. A.; Wang, J.; Hao, B.; Zhang, D.; Banyai, D.; Jaszczak, J. A.; Clark, K. W.; Idrobo, J. C.; Li, A. P.; Yap, Y. K. (2013). "Room-Temperature Tunneling Behavior of Boron Nitride Nanotubes Functionalized with Gold Quantum Dots". Advanced Materials. 25 (33): 4544-8. doi:10.1002/adma.201301339. PMID 23775671.

[12] Yu, J.; et al. (2006). "Isotopically Enriched 10BN Nanotubes" (PDF). Advanced Materials. 18 (16): 2157. doi:10.1002/adma.200600231. Archived from the original (PDF) on 2011-07-17.

[13] Ciofani, Gianni; Danti, Serena; Genchi, Giada Graziana; Mazzolai, Barbara; Mattoli, Virgilio (2013-05-27). "Boron Nitride Nanotubes: Biocompatibility and Potential Spill-Over in Nanomedicine". Small. 9 (9-10): 1672-85. doi:10.1002/smll.201201315. PMID 23423826.

[14] Zhong, J; Dai, L.C. (2012). "Targeting Liposomal Nanomedicine to Cancer Therapy". Technology in Cancer Research \& Treatment. 11 (5): 475-481. doi:10.7785/tcrt.2012.500259. PMID 22475065.

[15] Mortazavifar, A., Raissi, H., \& Akbari, A. (2018). DFT and MD investigations on the functionalized boron nitride nanotube as an effective drug delivery carrier for Carmustine anticancer drug. Journal of Molecular Liquids. doi:10.1016/j.molliq.2018.12.028.

[16] Ciofani, G. (2010). Potential applications of boron nitride nanotubes as drug delivery systems. Expert Opinion on Drug Delivery, 7(8), 889-893. doi:10.1517/17425247.2010.499897.

[17] Xu, H., Wang, Q., Fan, G., \& Chu, X. (2018). Theoretical study of boron nitride nanotubes as drug delivery vehicles of some anticancer drugs. Theoretical Chemistry Accounts, 137(7). doi:10.1007/s00214-018-2284-2.

[18] Shayan, K., \& Nowroozi, A. (2018). Boron nitride nanotubes for delivery of 5-fluorouracil as anticancer drug: a theoretical study. Applied Surface Science, 428, 500-513. doi:10.1016/j.apsusc.2017.09.121. 
[19] Khatti, Z., \& Hashemianzadeh, S. M. (2016). Boron nitride nanotube as a delivery system for platinum drugs: Drug encapsulation and diffusion coefficient prediction. European Journal of Pharmaceutical Sciences, 88, 291-297. doi:10.1016/j.ejps.2016.04.011.

[20] Liao, M.-L., Wang, Y.-C., Ju, S.-P., Lien, T.-W., \& Huang, L.-F. (2011). Deformation behaviors of an armchair boron-nitride nanotube under axial tensile strains. Journal of Applied Physics, 110(5), 054310. doi:10.1063/1.3626065.

[21] Yuan, J., \& Liew, K. M. (2010). Formation and Compressing Behavior of Coaxial Silicon Nanowires inside a Boron Nitride Nanotube. The Journal of Physical Chemistry C, 115(2), 431435. doi:10.1021/jp109210h.

[22] Panchal, M. B., \& Upadhyay, S. H. (2013). Doubly-Clamped Single Walled Boron Nitride Nanotube Based Nanomechanical Resonators: A Computational Investigation of Their Behavior. Journal of Nanotechnology in Engineering and Medicine, 3(4), 044501. doi:10.1115/1.4023897.

[23] Moon, W. H., \& Hwang, H. J. (2004). Molecular-dynamics simulation of structure and thermal behaviour of boron nitride nanotubes. Nanotechnology, 15(5), 431-434. doi:10.1088/0957-4484/15/5/005.

[24] Ghassemi, H. M., \& Yassar, R. S. (2010). On the Mechanical Behavior of Boron Nitride Nanotubes. Applied Mechanics Reviews, 63(2), 020804. doi:10.1115/1.4001117.

[25] Zheng, Y., Zhang, X., Nouri, M., Amini, A., Karimipour, A., Hekmatifar, M., ... Karimipour, A. (2020). Atomic rheology analysis of the external magnetic field effects on nanofluid in nonideal microchannel via molecular dynamic method. Journal of Thermal Analysis and Calorimetry. doi:10.1007/s10973-020-10191-2.

[26] Hekmatifar, M. Toghraie, D. Khosravi, A. Saberi F., Soltani, F. Sabetvand, R. Shahsavar Goldanlou A. (2020), The study of asphaltene desorption from the iron surface with molecular dynamics method, Journal of Molecular Liquids, 114325, 0167-7322. doi: 10.1016/j.molliq.2020.114325.

[27] Mosavi, A., Hekmatifar, M., Alizadeh, A., Toghraie, D., Sabetvand, R., Karimipour, A., (2020). The molecular dynamics simulation of thermal manner of $\mathrm{Ar} / \mathrm{Cu}$ nanofluid flow: The effects of spherical barriers size, Journal of Molecular Liquids, 114183. 
doi:10.1016/j.molliq.2020.114183.

[28] 8. Asgari, A., Nguyen, Q., Karimipour, A., Bach, Q.-V., Hekmatifar, M., \& Sabetvand, R. (2020). Investigation of additives nanoparticles and sphere barriers effects on the fluid flow inside a nanochannel impressed by an extrinsic electric field: A molecular dynamics simulation. Journal of Molecular Liquids, 114023. doi:10.1016/j.molliq.2020.114023.

[29] Qiu, H., Shen, R., \& Guo, W. (2010). Vibrating carbon nanotubes as water pumps. Nano Research, 4(3), 284-289. doi:10.1007/s12274-010-0080-y.

[30] Lohrasebi, A., \& Jamali, Y. (2011). Computational modeling of a rotary nanopump. Journal of Molecular Graphics and Modelling, 29(8), 1025-1029. doi:10.1016/j.jmgm.2011.04.007.

[31] Plimpton, S. J., \& Thompson, A. P. (2012). Computational aspects of many-body potentials. MRS Bulletin, 37(05), 513-521.

[32] Brown, W. M., Wang, P., Plimpton, S. J., \& Tharrington, A. N. (2011). Implementing molecular dynamics on hybrid high-performance computers - short-range forces. Computer Physics Communications, 182(4), 898-911.

[33] Mai, W.; Li, P.; Bao, H.; Li, X.; Jiang, L.; Hu, J.; Werner, D. H. (April 2019). "Prism-Based DGTD With a Simplified Periodic Boundary Condition to Analyze FSS With D2n Symmetry in a Rectangular Array Under Normal Incidence". IEEE Antennas and Wireless Propagation Letters. 18 (4): 771-775. doi:10.1109/LAWP.2019.2902340. ISSN 1536-1225.

[34] Nosé, S (1984). "A unified formulation of the constant temperature molecular-dynamics methods" (PDF). Journal of Chemical Physics. 81 (1): 511-519. Bibcode:1984JChPh..81..511N. doi:10.1063/1.447334.

[35] Hoover, William G. (Mar 1985). "Canonical dynamics: Equilibrium phase-space distributions". Phys. Rev. A. 31 (3): 1695-1697. Bibcode:1985PhRvA..31.1695H. doi:10.1103/PhysRevA.31.1695. PMID 9895674.

[36] Tersoff, J. (1988). New empirical approach for the structure and energy of covalent systems. Physical Review B, 37(12), 6991-7000. doi:10.1103/physrevb.37.6991.

[37] Tersoff, J. (1989). Modeling solid-state chemistry: Interatomic potentials for multicomponent 
systems. Physical Review B, 39(8), 5566-5568. doi:10.1103/physrevb.39.5566.

[38] Daw, Murray S.; Stephen Foiles (1993). "The embedded-atom method: a review of theory and applications". Mat. Sci. Eng. Rep. 9 (7-8): 251. doi:10.1016/0920-2307(93)90001-U.

[39] Daw, Murray S.; Mike Baskes (1984). "Embedded-atom method: Derivation and application to impurities, surfaces, and other defects in metals". Physical Review B. American Physical Society. 29 (12): 6443-6453. Bibcode:1984PhRvB..29.6443D. doi:10.1103/PhysRevB.29.6443.

[40] Mayo, S. L., Olafson, B. D., \& Goddard, W. A. (1990). DREIDING: a generic force field for molecular simulations. The Journal of Physical Chemistry, 94(26), 8897-8909. doi:10.1021/j100389a010.

[41] Rapaport, D. C. (2004). The Art of Molecular Dynamics Simulation. doi:10.1017/cbo9780511816581.

[42] Hairer, Ernst; Lubich, Christian; Wanner, Gerhard (2003). "Geometric numerical integration illustrated by the Störmer/Verlet method". Acta Numerica. 12: 399-450. Bibcode:2003AcNum..12..399H. CiteSeerX 10.1.1.7.7106.

[43] Swope, William C.; H. C. Andersen; P. H. Berens; K. R. Wilson (1 January 1982). "A computer simulation method for the calculation of equilibrium constants for the formation of physical clusters of molecules: Application to small water clusters". The Journal of Chemical Physics. 76 (1): 648 (Appendix). Bibcode:1982JChPh..76..637S. doi:10.1063/1.442716.

[44] Insepov, Z., Wolf, D., \& Hassanein, A. (2006). Nanopumping Using Carbon Nanotubes. Nano Letters, 6(9), 1893-1895. doi:10.1021/n1060932m.

[45] Chen, M., Zang, J., Xiao, D., Zhang, C., \& Liu, F. (2009). Nanopumping molecules via a carbon nanotube. Nano Research, 2(12), 938-944. doi:10.1007/s12274-009-9096-6. 